\title{
Percepción de la imagen corporal de los adolescentes de Huelva atendiendo al género y a la edad Body image perception by gender and age in adolescents of Huelva Manuel Javier Arrayás Grajera, Inmaculada Tornero Quiñones, Martín Salvador Díaz Bento Universidad de Huelva (España)
}

Resumen. El objetivo principal de este estudio es comprobar las diferencias entre la percepción de la imagen corporal (en adelante IC) en adolescentes de Huelva atendiendo tanto al género como a la edad. La muestra estuvo compuesta por 226 estudiantes, con edades comprendidas entre 12 y 18 años (M=13,94; DT=1,41). La distribución por sexo fue de 54,9\% de chicos ( $n=124)$ y 45,1\% de chicas ( $n=102)$. Los instrumentos que se utilizaron para evaluar los diferentes componentes de la IC fueron: 1) Para evaluar el componente perceptual se utilizó el método de estimación corporal global de Gardner, Stark, Jackson y Friedman (1999), adaptado al español por Rodríguez, Beato, Rodríguez \& Martínez (2003). 2) Para evaluar el componente subjetivo se utilizó la subescala de insatisfacción corporal del Eating Disorders Inventory (EDI) de Garner, Polivy \& Olmstead (1983), adaptado a la población española por Garner (1998). 3) Para evaluar el componente conductual se utilizó el Body Image Avoidance Questionnaire (BIAQ,), de Rosen, Salzberg, Srebnik \& Went (1990). Las chicas se sienten más insatisfechas con su IC que los chicos. La mayoría de chicos y chicas coinciden en el deseo de perder peso. Las chicas mostraron un mayor uso de conductas de evitación a causa de la IC que los chicos especialmente en el «modo de llevar la ropa», «las actividades sociales» y «pesarse y acicalarse». Las chicas mostraron mayor «Obsesión por la delgadez» que los chicos.

Palabras clave. Imagen corporal, Adolescentes, género, edad.

Abstract. The main objective of this study is to assess differences in body image (from now on BI) perception among Huelva teenagers, based on gender and age. The sample comprised 226 students aged between 12 and 18 years $(\mathrm{M}=13.94$, SD = 1.41). Gender distribution was $54.9 \%$ boys ( $=$ $124)$ and $45.1 \%$ girls $(n=102)$. The instruments used to assess the different components of the BI were: 1$)$ Global corporal estimating method of Gardner, Stark, Jackson and Friedman (1999) to evaluate the perceptual component, adapted to Spanish by Rodriguez, Beato, Rodriguez and Martinez (2003); 2) Body dissatisfaction subscale of the Eating Disorder Inventory (EDI) of Garner, Polivy \& Olmstead (1983) to assess the subjective component, adapted to the Spanish population by Garner (1998); 3) Body Image Avoidance Questionnaire (BIAQ) of Rosen, Salzberg, Srebnik \& Went (1990) to evaluate the behavioral component. Girls are more dissatisfied with their BI than boys. Most boys and girls have similar desire to lose weight. Girls showed greater use of avoidance behaviors due to BI than boys, especially in the «how to wear clothes», «social activities», and «weighed and groom» subscales. Girls showed greater «obsession with thinness» than boys.

Keywords. Body image, adolescents, gender, age.

\section{Introducción}

En nuestros días, existe una creciente preocupación de la sociedad por la adquisición de una IC acorde a los cánones estéticos de moda. Estamos asistiendo por tanto a una verdadera revolución de la estética, que se postula como un fuerte mediador de la vida social, convirtiéndose la IC en preocupación, ya que llega a ser un elemento de clara discriminación social (Toro, 1996).

Actualmente, son muchos los estudios (Duncan,Al-Nakeeb, Nevill \& Jones, 2004; Camacho, Fernández \& Rodríguez, 2006; Slater \& Tiggemann, 2006; Tornero \& Sierra, 2008; Urrutia, Azpillaga, Cos \& Muñoz, 2010) que utilizan la IC como contenido, pero en todos ellos, esta IC se ha definido de diferente manera en función de la perspectiva bajo la que se ha realizado tal estudio y se han utilizado diferentes términos: esquema corporal, modelo postural, yo corporal, concepto corporal, etc.

En la literatura científica encontramos multitud de definiciones acerca del término de IC, por lo tanto, es preciso consensuar una definición de lo que entendemos por IC. La definición más clásica la describe como una imagen desarrollada por nuestra mente sobre nuestro propio cuerpo (Schilder, 1935). De forma más dinámica y actual, Raich (2001), la define en términos de sentimientos y actitudes hacia el propio cuerpo.

El constructo de IC es considerado actualmente desde una perspectiva multidimensional que engloba percepciones, pensamientos, sentimientos, actitudes y comportamientos referentes al propio cuerpo y cuyas alteraciones pueden dar lugar a problemas emocionales (Cash \& Pruzinsky, 1990; Grogan, 1999; Thompson, 2004; Maganto \& Cruz, 2008)

No obstante, cuando se habla de IC, no lo hacemos de un concepto concreto, sino que está compuesto por diferentes componentes. Son muchos los autores que conciben el constructo IC constituido por tres componentes (Grogan, 1999; Lox, Martín \& Petruzzello, 2003; Markham, Thompson \& Bowling, 2005; Salaberría, Rodríguez \& Cruz,

Fecha recepción: 23-08-16. Fecha de aceptación: 20-09-17 Manuel Javier Arrayás Grajera

manueljavier.grajera@dempc.uhu.es
2007): a) Un componente perceptual: que hace referencia a la precisión con que se percibe el tamaño corporal de diferentes segmentos corporales o del cuerpo en su totalidad. b) Un componente subjetivo (cognitivoafectivo): que atiende a las actitudes, sentimientos, y valoraciones que despierta el cuerpo, principalmente el tamaño corporal, el peso, las partes del cuerpo o cualquier otro aspecto de la apariencia física. c) Un componente conductual: en clara referencia a las conductas que la percepción del cuerpo y sentimientos asociados provocan.

La IC, al ser un constructo tan complejo y arraigado en la persona tiene complejas relaciones con otros conceptos de importancia como el género y la edad

Con respecto al género, podemos decir que la insatisfacción con la IC puede ocurrir tanto en hombres como en mujeres, de hecho investigaciones más recientes como la de Cash (2004), se ha expandido para incluir a personas de ambos géneros. Sin embargo, la mayoría de los estudios se han centrado en las mujeres ya que este grupo ha mostrado mayores dificultades con la insatisfacción corporal (McCabe \& Ricciardelli, 2001). No obstante, aunque los varones adolescentes tienden a estar más satisfechos con su cuerpo (Ureña, Ramos y Salas, 2015; González, Cuervo, Cachón y Zagalaz, 2016), tanto estos y otros estudios han indicado que el trastorno de la IC es cada vez mayor entre los hombres, siendo la dismorfia muscular, un trastorno más probable en hombre que en mujeres (Pope, Gruber, Choi, Olivardia \& Phillips, 1997).

Con respecto a la edad, existe cierta discrepancia, ya que varios estudios han indicado que la alteración de la IC aumenta con la edad (Demarest \& Allen, 2000), mientras que otros han informado de que la edad tiene poco efecto (Cash \& Henry, 1995).

En definitiva, la IC es un constructo complejo que incluye tanto la percepción que tenemos del cuerpo y de cada una de sus partes, como del movimiento y los límites de éste, la experiencia subjetiva de actitudes, pensamientos, sentimientos y valoraciones que hacemos y sentimos y el modo de comportarnos derivado de las cogniciones y sentimientos que experimentamos (Raich, 2001).

Hoy día está ampliamente aceptado que las causas de las alteraciones de la IC no se pueden asociar a un único factor, sino que pueden estar provocadas por diferentes factores como la familia, la cultura, las relaciones interpersonales, por características personales como el géne- 
ro, la personalidad, los aspectos físico-biológicos, la actividad física, entre otros. Determinar y analizar las causas de esas alteraciones es fundamental ya que nos ayudará a entender mejor dichos trastornos proporcionándonos información útil para llevar a cabo una intervención eficaz. Por todo ello, el objetivo principal de este estudio es comprobar las diferencias entre la percepción de la imagen corporal en adolescentes de Huelva atendiendo tanto al género como a la edad.

\section{Metodología}

\section{Participantes}

La muestra empleada está compuesta por 226 estudiantes de educación secundaria obligatoria de Huelva con edades comprendidas entre 12 y 18 años ( $\mathrm{M}=13,94 ; \mathrm{DE}=1,41)$. La distribución porcentual por sexo del alumnado fue $54,9 \%$ para los chicos $(n=124)$ y $45,1 \%$ para las chicas ( $n=102)$. Siguiendo a Heinemann (2008), los sujetos han sido elegidos por un muestreo probabilístico estratificado por conglomerados, puesto que aunque la muestra sea analizada en su totalidad, se han formado subgrupos determinados por el criterio «curso académico». Se ha tenido en cuenta al total de alumnos que se encontraban en clase, sin exclusión deningún tipo

\section{Variables e instrumentos}

Las variables que se analizaron en este estudio fueron las siguientes:

- Género y edad.

- Percepción de la IC (satisfacción/insatisfacción corporal).

- Subjetividad de la IC (subescala de insatisfacción corporal).

- Conductas de la IC (frecuencia de las conductas de evitación).

Para medir el componente perceptual de la IC se utilizó el método de estimación corporal global de Gardner, Stark, Jackson \& Friedman, (1999), adaptadas al español por Rodríguez, Beato, Rodríguez \& Martínez (2003). La fiabilidad interna de estas escalas fue de á $=0,71$.

Se tomó la escala de valoración de la imagen corporal de Gardner, Stark, Jackson \& Friedman (1999) para su uso. En el formato original, dicha escala, está formada por 13 siluetas de $8 \mathrm{~cm}$ de altitud, que representan contornos esquemáticos de la figura humana, en ausencia de cualquier atributo como pelo o rostro. Las siluetas se elaboraron atendiendo a estadísticas del Centro Nacional de la Salud de los Estados Unidos para una edad media de 19 años y un peso de 63,99 kg (desviación típica [DT]=14,53). La figura media representa la mediana de la distribución de peso para la población de referencia; sobre dicha silueta media se hicieron modificaciones que incrementaban o disminuían \pm $30 \%$ su volumen, para contruir un total de seis siluetas más que representaran en orden creciente el incremento de peso (5\%, 10\%, 15\%, $20 \%$, 25\% y 30\%), así como aquellas seis siluetas que representan proporcionalmente la progresiva disminución de peso $(-5 \%,-10 \%$, $15 \%,-20 \%,-25 \%$ y $-30 \%)$; con ese sumativo de siluetas se produce un continuo de siluetas, cuyos máximo y mínimo representan una figura extremamente delgada y otra obesa.

La escala mencionada permite la obtención de tres índices: uno representa la percepción actual de su imagen corporal; el segundo muestra, la imagen que los usuarios estiman como «ideal» para si mismos. Por último, el evaluador señala la imagen real que muestra cada una de los individuos evaluados. De este modo se puede evaluar el grado de ajuste entre la imagen percibida y la deseada, así como una estimación de la imagen corporal objetiva realizada por el evaluador (para este estudio, solo se tomaron en cuenta la imagen percibida y deseada).

Para medir el componente subjetivo de la IC se empleó el Eating Disorders Inventory (EDI) de Garner, Polivy \& Olmstead (1983), adaptado a la población española por Garner (1998). El cuestionario mencionado mide anorexia y bulimia nerviosa, en su formato original se compone de 64 preguntas que se agrupan en 8 subescalas. Las subescalas que se utilizaron para este estudio fueron la obsesión por la delgadez, la bulimia y la insatisfacción corporal, que miden actitudes y comportamientos relacionados con la alimentación, el peso y la forma corporal. De acuerdo con el estudio de Gardner, et al. (1983), la subescala obsesión por la delgadez mide preocupación con el peso y una extrema preocupación con la alimentación. La subescala bulimia mide la presencia de episodios de grandes ingestas alimenticias con la posterior acción de deseo de autoproducirse el vómito. La subescala insatisfacción corporal examina si un individuo está insatisfecho con la forma de su cuerpo y considera que alguna, o más de una, parte de su cuerpo es demasiado grande o demasiado gorda. La fiabilidad interna de estas subescalas fueron á $=0,84$, á $=0,72$ y á $=0,74$ respectivamente.

Para medir el componente conductual de la IC se utilizó el Body Image Avoidance Questionnaire (BIAQ), cuestionario que evalúa la frecuencia de conductas de evitación relacionadas con la IC de Rosen, Salzberg, Srebnik \& Went, (1990). Consta de 19 ítems en los que se evalúa la frecuencia con la que se practican conductas que denotan cierta preocupación por la apariencia física y conductas de evitación. Contiene un total de cuatro subescalas: vestimenta, actividades sociales, restricción alimentaria y pesarse y acicalarse. También puede valorarse la puntuación de todos los ítems. La fiabilidad interna de todos los ítems del BIAQ fue á = 0,72.

\section{Diseñoy procedimiento}

Se trata de un estudio de tipo social, transversal, descriptivo y correlacional (Tamayo y Tamayo, 1999).

En primer lugar se contactó con los centros y se mantuvo reuniones informativas con los respectivos directores. Tras contar con el consentimiento de los directores, nos entrevistamos con los profesores de Educación Física a los cuales se informó convenientemente solicitando su colaboración. Hecho esto, se solicitaron los permisos por escrito tanto a la dirección de los centros, como del profesorado implicado y se procedió a solicitar la autorización de los padres/madres de los participantes por ser menores de edad.

El día acordado por el centro, los profesores de Educación Física y los investigadores, administraron los cuestionarios. Estos se llevaron a cabo durante la clase de educación física, dando las instrucciones necesarias para su cumplimentación, insistiendo los investigadores en el carácter anónimo y voluntario del mismo. Así mismo, se hizo hincapié en la necesidad de ser sinceros en las respuestas. La cumplimentación del cuestionario dura aproximadamente 45 minutos.

\section{Análisis Estadístico}

Para realizar los cálculos estadísticos aplicados en el presente estudio se utilizó el programa de estadística Statistical Package for Social Sciences (SPSS), versión 19.0. Para todas las pruebas estadísticas se usó un nivel de significación de .05. Los análisis realizados a los datos obtenidos han sido:

a) Estadística descriptiva univariable(Sánchez \& Carmona, 2004): se utilizaron tablas de frecuencias y sus respectivas medias y desviaciones típicas.

b) Estadística descriptiva bivariable (Sánchez \& Carmona, 2004):

- Dos variables cualitativas: se utilizaron tablas de contingencias para describir numéricamente la relación entre ambas.

- Dos variables cuantitativas: se utilizaron los coeficientes de correlación de Pearson para calcular el índice que nos informe de cuán relacionadas están las dos variables.

- Una variable cuantitativa y otra cualitativa: se utilizó la comparación de medias por medio de la prueba $T$ para muestras independientes.

\section{Resultados}

Imagen corporal percibida, deseada e insatisfacción corporal atendiendo al género y la edad

En la tabla 1, se observa como las chicas de este estudio, mostraron una percepción de su IC menos voluminosa que los chicos ( $p=.008$ ), a pesar de lo cual, su IC deseada obtuvo puntuaciones más altas ( $p<$ .001), de ahí que la insatisfacción de estas fuera significativamente mayor que en el caso de los chicos $(p=.001)$. A pesar de todo lo anterior, las diferencias en el grado de insatisfacción (satisfechas, insatisfechas tipo I: deseo perder peso, insatisfechas tipo II: deseo ganar peso), no son significativas entre ambos géneros ( $p=.636)$. 
En la tabla 2, vemos como el mayor porcentaje de chicos y chicas se encuentra en el tipo II de insatisfacción: deseo perder peso (57\% y $69,6 \%$ respectivamente). Esto significa que prácticamente dos de cada cinco adolescentes varones y casi tres de cada cuatro adolescentes mujeres están desconformes con su IC y tienen el deseo de perder peso.

Cuando analizamos la imagen corporal percibida, la imagen corporal deseada, la insatisfacción corporal, el tipo y el grado de insatisfacción corporal atendiendo a la edad no encontramos para nuestro estudio diferencias estadísticamente significativas ni en chicos ni en chicas.

Diferencias de género con respecto a las subescalas del EDI (insatisfacción corporal, bulimia y obsesión por la delgadez)

Los adolescentes de este estudio, mostraron mayor puntuación que las adolescentes de este estudio en las dimensiones del EDI «Insatisfacción corporal» $\mathrm{y}$ «Bulimia», siendo estas diferencias poco o nada significativas ( $p=0.38$ y $p=.163$, respectivamente). Por el contrario, las chicas mostraron mayor «Obsesión por la delgadez» siendo en este caso la diferencias significativas $(p<.001)$, (Tabla 4).

$\mathrm{Al}$ analizar cada una de las subescalas del EDI no encontramos diferencias estadísticamente significativas ni en el caso de los chicos ni el de las chicas.

Diferencias de género con respecto a las dimensiones del BIAQ

Las adolescentes de este estudio, mostraron mayor puntuación que los adolescentes de este estudio en todas las dimensiones del BIAQ: «puntuación total del BIAQ» $(p<.001)$, «modo de llevar la ropa» $(p=$ $.006)$, «actividades sociales» $(p=.005)$, «restricción de comida» ( $p=$ $.634) \mathrm{y}$ «pesarse y acicalarse» $(p<.001)$. En todas ellas las diferencias fueron significativas excepto para la dimensión «restricción de comida» (Tabla 3).

Cuando analizamos todas las dimensiones del BIAQ y la puntuación global del mismo, observamos como a medida que aumenta la edad, no se producen diferencias estadísticamente significativas, solo en el caso de las chicas, presentan un aumentos significativo de la dimensión «pesarse y acicalarse» a medida que aumenta la edad $(\mathrm{r}=453 ; p<.001)$.

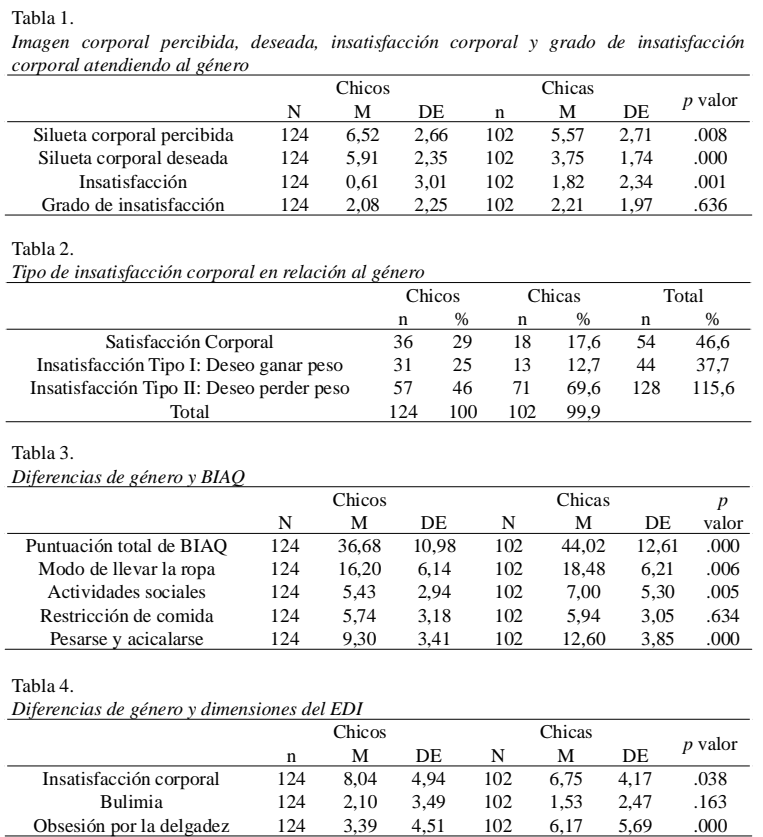

\section{Discusión}

Atendiendo a los resultados respecto al tipo y al grado de insatisfacción corporal, comprobamos como los valores entre la muestra masculina y femenina difieren notablemente en cuanto a la IC percibida, deseada y a la satisfacción con dicha IC. Casualmente coinciden en el tipo de insatisfacción por un aspecto negativo, ambos sexos están insatisfechos por el deseo de perder peso. En el estudio de Ramos,
Rivera \& Moreno (2010), en el que examinaron las diferencias entre 21.811 chicos y chicas adolescentes (11-18 años), se pone de manifiesto como a pesar de que las chicas tenían una puntuación en el Índice de Masa Corporal (IMC) más ajustada y presentaban un nivel menor de sobrepeso y obesidad, estaban más insatisfechas con su IC, corroborando los resultados observados en los estudios de Ureña, Ramos \& Salas, (2015) y González, Cuervo, Cachón \& Zagalaz, (2016). En esta misma línea, los autores afirman que la razón que mueve a chicos y chicas a realizar dietas para adelgazar no es el peso corporal real, sino la percepción que tienen de su cuerpo, en primer lugar, y cómo de satisfechos están con él, en segundo lugar. Estos resultados no concuerdan con los obtenidos por Marrodán, et al. (2008) y Mendo, Polo, Amado, Iglesias \& León (2017) que analizaron la apreciación de la imagen durante la adolescencia y la niñez, respectivamente. Estos autores puntualizan que la disconformidad muestra distinta tendencia de género de manera que, mientras la mayor parte de las chicas quisieran tener una silueta más estilizada, por lo general los varones desearían ser algo más robustos, en nuestro caso la mayoría de varones desearían perder peso.

Haciendo referencia a la IC en su conjunto, tal como mencionan Matos, Simões, Camacho, Reis \& Equipa Aventura Social (2014) en el estudio Health Behaviour in School-aged Children (HBSC), «A saúde dos adolescentes portugueses», en el cual se concluye que tanto los varones como los estudiantes más jóvenes son los que presentan mayor satisfacción con su IC. Por el contrario, las mujeres y los discentes de más edad, cambiarían algo en su cuerpo y afirman estar siguiendo en la actualidad una dieta. Este trabajo apunta que la mayoría de los estudiantes piensan que tienen un aspecto normal en referencia a su apariencia física.

Las adolescentes de este estudio, mostraron mayor puntuación que los adolescentes de este estudio en todas las dimensiones del BIAQ («puntuación total del BIAQ», «modo de llevar la ropa», «actividades sociales», «restricción de comida» y «pesarse y acicalarse». En todas ellas las diferencias fueron significativas excepto para la dimensión «restricción de comida». El estudio de Harris \& Carr (2001), pone de manifiesto que la preocupación por el aspecto físico tiene una gran incidencia en los sujetos sean hombres o mujeres. Este control del peso en pro de evitar enfermedades como la obesidad, ya fue apuntado por Melo \& Venâncio (2004), quienes manifestaron que la obesidad y su correspondiente desajuste de la IC es consecuencia del estilo de vida moderno entre otros factores.

Aunque los adolescentes de este estudio mostraron más «insatisfacción corporal» que las chicas, fueron estas las que obtuvieron un resultado significativamente mayor en cuanto a la «Obsesión por la delgadez». En la literatura se hallan resultados similares, sobre todo en relación a la subescala obsesión por la delgadez. Fan, et al. (2010) hallaron que las chicas tenían puntuaciones significativamente más altas en las dos subescalas del EDI que los chicos. De la misma manera concluyen estudios como los de Rodgers, Paxton \& Chabrol (2010), Rodgers, Faure \& Chabrol (2009), Urzúa, Castro, Lillo \& Leal (2009), y Aliaga-Deza, De la Cruz-Saldaña \& Vega-Dienstmaier (2010) en los que las mujeres tienden a mostrar mayor obsesión por la delgadez y bulimia que los varones, aunque las diferencias en la subescala bulimia no fueron en estos estudios estadísticamente significativas.

\section{Conclusiones}

A través del estudio realizado para analizar la percepción de la IC de los adolescentes de Huelva atendiendo al género y a la edad concluimos que las chicas son las que más insatisfechas se sienten con su IC en comparación con los chicos.

Del mismo modo, el grupo femenino del estudio muestra un mayor uso de conductas de evitación que los chicos a causa de la IC, especialmente en el «modo de llevar la ropa», «las actividades sociales» y «pesarse y acicalarse». Además de, las chicas, mostrar una mayor «obsesión por la delgadez» que la mostrada por los chicos en el estudio.

Por otro lado, cabe mencionar que tanto chicos como chicas son coincidentes en el deseo de perder peso. 


\section{Referencias}

Aliaga-Deza, L., De la Cruz-Saldaña, T.A., \& Vega-Dienstmaier, J.M. (2010). Sintomatología de los trastornos de la conducta alimentaria en adolescentes de un colegio del distrito de Independencia, Revista Neuro Psiquiatría, 73, 52-61

Camacho, M.J., Fernández, E., \& Rodríguez, M.I. (2006). Imagen corporal y práctica de actividad física en las chicas adolescentes: Incidencia de la modalidad deportiva. International Journal of Sport Science, 2(3), 1-19.

Cash, T.F., \& Henry, P.E. (1995). Women’s body images: The results of a national survey in the U.S.A. Sex Roles, 33, 19-28.

Cash, T.F., \& Pruzinsky, T. (Eds.). (1990). Body Images: Development, Deviance, and Change. Nueva York: Guilford Press.

Cash, T.F. (2004). Body image: past, present and future. Body Image, $1,1-5$.

Demarest, J., \& Allen, R. (2000). Body image: Gender, ethnic, and age differences. Journal of Social Psychology, 140(4), 465-472.

Duncan, M.J., Al-Nakeeb, Y., Nevill, A., \& Jones, M.V. (2004). Body image and physical activity in British secondary school children. European Physical Education Review, 10, 243-260.

Fan, Y., Lit, Y., Liu, A., Hu, X., Ma, G, \& Xu, G. (2010). Associations between body mass index, weight control concerns and behaviors, and eating disorder symptoms among non-clinical Chinese adolescents. BMC Public Health, 314(10).

Garner, D.M., Polivy, J., \& Olmstead, M.P. (1983). Development and validation of a multidimensaional eating disorder inventory for anorexia nervosa and bulimia. International Journal of Psychiatry in Medicine, 11, 263-284.

Garner, D.M. (1998). EDI 2. Inventario de trastornos de la conducta alimentaria. Madrid: Tea Ediciones.

Gardner, R.M., Stark, K., Jackson, N., \& Friedman, B.N. (1999). Development and validation of two new scales for assessment of body-image. Perceptual Motor Skills, 87, 981-983.

González, C., Cuervo, C., Cachón, J., \& Zagalaz, M. L. (2016). Relación entre variables demográficas, la práctica de ejercicio físico y la percepción de la imagen corporal en estudiantes del grado de magisterio. Retos: Nuevas tendencias en educación física, deporte y recreación, 29, 90-94.

Grogan, S. (1999). Body image: understanding body dissatisfaction in men, women and children. London: Routledge

Harris, D.L., \& Carr,A.T. (2001). Prevalence of concern about physical appearance in the general population. British Journal of Plastic Surgery, 54(3), 223-226.

Heinemann, K. (2008). Introducción a la metodología de la investigación empírica en las ciencias del deporte. Badalona: Paidotribo.

Ivarsson, T., Svalander, P., Litlere, O., \& Nevonen, L. (2006). Weight concerns, body image, depression and anxiety in Swedish adolescents. Eating Behaviors, 7(2), 161-175.

Law, C., \& Peixoto, M. (2002). Cultural standards of attractiveness: A thirty year look at changes in male images in magazines. Journalism and Mass Communication Quarterly, 79(3), 697-711.

Lox, C. L., Martin, K. A., \& Petruzzello, S. J. (2003). The psychology of exercise: Integrating theory and practice. Scottsdale,AZ: Holcomb Hathaway.

Maganto, C. \& Cruz, S. (2008). TSA. Test de Siluetas para Adolescentes. Madrid: TEA Ediciones.

Markham, A., Thompson, T., \& Bowling, A. (2005). Determinants of body-image shame. Personality and Individual Differences, 38, 1529-1541.

Marrodán, M.D., Montero-Roblas, V., Mesa, M.S., Pacheco, J.L., González, M., Bejarano, I., Lomaglio, D.B., Verón, J.A., \& Carmenate, M. (2008). Realidad, percepción y atractivo de la imagen corporal: condicionantes biológicos y socioculturales. Zainak, 30, 15-28.

Matos, M. G., Simões, C., Carvalhosa, S. F., Reis, C., \& Canha, L. A. (1998). Asaúde dos adolescentes portugueses. Estudo Nacional da
Rede Europeia HBSC/OMS. Aventura Social \& Saúde. Lisboa: Falcultade da Motricidade Humana.

Matos, M. G.., Simões, C., Camacho, I., Reis, M., \& Equipa Aventura Social. (2014). A saúde dos adolescentes portugueses. Relatorio do Estudo HBSC 2014. Aventura Social \& Saúde. Lisboa: Falcultade da Motricidade Humana.

McCabe, M.P., \& Ricciardelli, L.A. (2001). Parent, peer, and media influences on body image and strategies to both increase and decrease body size among adolescent boys and girls. Adolescence, 36, 225240.

Melo, \& Venâncio (2004). Atividade física e saúde: discursos que controlam o corpo. Pensar a prática, 7(1), 59-74.

Mendo, S., Polo, M. I., Amado, D., Iglesias, D., \& León, B. (2017). Selfconcept in childhood: The role of body image and sport practice. Frontiers in psychology, 8: 853

Pope, H.G., Gruber, A.J., Choi, P., Olivardia, R., \& Phillips, K.E. (1997). Muscle dysmorphia: An underecognized form of body dysmorphic disorder. Psychosomatics, 38, 548-557.

Raich, R.M. (2001). Imagen corporal. Conocer y valorar el propio cuerpo. Madrid: Editorial Pirámide.

Ramos, P., Rivera, F., \& Moreno, C. (2010). Diferencias de sexo en imagen corporal, control de peso, e índice de masa corporal de los adolescentes españoles. Psicothema, 22 (1), 77-83.

Rodgers, R. F., Faure, K., \& Chabrol, H. (2009). Gender differences in parental influences on adolescent body dissatisfaction and disordered eating. Sex Roles, 61(11-12), 837-849.

Rodgers, R.F., Paxton, S.J., \& Chabrol, H. (2010). Depression as a moderator of sociocultural influences on eating disorder symptoms in adolescent females and males. Journal of Youth Adolescents, 39(4), 393-402.

Rodríguez, M.A., Beato, L., Rodríguez, T., \& Martínez-Sánchez, F. (2003).Adaptación española de la escala de evaluación de la imagen corporal de Gardner en pacientes con trastornos de la conducta alimentaria. Actas Españolas de Psiquiatría, 31(2), 59-64.

Rosen, J.C., Srebnik, D., Saltzberg, E., \&Wendt, S. (1990). Development of a body avoidance questionnaire. Psychological Assessment: A Journal of Consulting and Clinical Psychology, 3(1), 32-37.

Salaberría, K., Rodríguez, S., \& Cruz, S. (2007). Percepción de la imagen corporal. Osasunaz, 8, 171-183.

Sánchez, M., \& Carmona, J. (2004). Análisis de datos con SPSS 12. Huelva: Universidad de Huelva.

Schilder, P. (1935). Image and appearance of the human body. Londres: Kegan Paul, Trench Trubner and Co.

Slater, A., \& Tiggemann, M. (2006). The Contribution of Physical Activity and Media Use during Childhood and Adolescence to Adult Women's Body Image. Journal of Health Psychology, 11(4), 553-565.

Tamayo y Tamayo, M. (1999). La investigación. En Martínez, P. (Coor.), Aprender a investigar. ICFES: Santa Fe de Bogotá, D.C.

Thompson, J. (2004). Handbook of eating disorders and obesity. New York, NY: Wiley.

Tornero, I., \& Sierra, Á. (2008). Satisfacción corporal y actividad física en el alumnado de la facultad de ciencias de la educación de la Universidad de Huelva. Congreso Internacional y XXV Nacional de Educación Física, 4, 1-4. Córdoba. Universidad de Córdoba.

Toro, J. (1996). El cuerpo como delito: anorexia, bulimia, cultura y sociedad. Barcelona: Ariel.

Ureña, P., Blanco, L., \& Salas, J. (2015). Calidad de Vida, indicadores antropométricos y satisfacción corporal en un grupo de jóvenes colegiales. Retos: Nuevas tendencias en educación física, deportey recreación, 27, 62-66.

Urrutia, S., Azpillaga, I., de Cos, G.L., \& Muñoz, D. (2010). Relación entre la percepción de estado de salud con la práctica Físico-deportiva y la imagen Corporal en adolescentes. Cuadernos de Psicología del Deporte, 10(Sup), 51-56.

Urzúa, A., Castro, S., Lillo, A., \& Leal, P. (2009). Evaluación de los trastornos alimentarios: propiedades psicométricas del 